\title{
A Bicistronic DNA vaccine against Porcine Circovirus and Porcine Parvovirus
}

\section{Wencheng Lin", Zhenmei Zhang" and Shangjin Cui*}

Division of Swine infectious Disease, State Key Laboratory of Veterinary Biotechnology, Harbin Veterinary Research Institute, Harbin 150001, China \#These authors contributed equally to the authorship of this work

\begin{abstract}
Background: Porcine parvovirus (PPV) is a widespread, infectious virus associated with reproductive disease of swine and death of piglets, and porcine circovirus type 2 (PCV2) is an important emerging pathogen associated with a number of different syndromes and diseases in pigs.
\end{abstract}

Objectives: The aim of this study was to construct a series of recombinant plasmids used for immunizing mice and measure the immune responses.

Methods: A bicistronic DNA vaccine against PPV and PCV2 infection was developed by subcloning PPV-VP2 and PCV2-ORF2 genes into a bicistronic vector. After the in vitro expression of both the proteins was characterized, the bicistronic DNA vaccine and monocistronic DNA vaccines were injected into mice and the induced immune responses were compared with monocistronic DNA vaccines.

Results: There was no significant difference in ELISA antibody, virus neutralizing antibody and cellular immune responses against PPV and PCV2 in mice immunized with bicistronic or monocistronic DNA vaccine, respectively.

Conclusions: This study indicates that bicistronic DNA vaccine can induce humoral and cellular immune responses in mice against both PPV and PCV2.

Keywords: Bicistronic DNA vaccine; Immunization; Immune responses; Mice; Porcine circovirus; Porcine parvovirus

\section{Introduction}

Porcine circovirus 2 (PCV2) was first recognized in 1996, when an infection was identified in specific-pathogen-free swine herds in western Canada. The disease caused by PCV2 was reported as a new syndrome, termed post-weaning multisystemic wasting syndrome (PMWS) [1,2]. Currently, PCV2 is considered to be an important emerging pathogen associated with a number of different syndromes and diseases in pigs, which have been collectively named porcine circovirus diseases (PCVD) [3].

Another important pathogen, porcine parvovirus (PPV), is a major cause of maternal reproductive failure of swine [4]. In contrast to the generally accepted concept of PPV pathogenesis in reproductive failure, PPV/PCV2 co-infections have been demonstrated in a significant portion of field cases of PMWS in Korea [5,6] and Canada [7]. Coinfection with PCV2 and PPV induces more severe lesions and clinical disease than infections by either pathogen alone [8,9]. The clinical manifestations associated with co-infection or singular infection by these two viruses include many diseases, and outbreaks of these two viruses have resulted in substantial economic loss. No effective antiviral drugs for PCV2 or PPV are available.

After the concept of DNA immunization was developed in 1990 $[10,11]$, the method has developed rapidly. Experimental applications of DNA vaccines have been tested against a large number of viral, bacterial, and parasitic diseases using not only intramuscular but also intradermal, mucosal, oral, and biojector inoculations or direct skin delivery $[12,13]$. DNA vaccines could be especially useful for developing countries because unlike conventional vaccines, DNA vaccines do not require cold chain storage and transportation, are easy to manufacture and are therefore economical, and provide long-lasting immune responses.
Use of multivalent vaccines for immunization against different viral and bacterial diseases is common for animals including dogs [10], swines. PCV2 and PPV could cause coinfection, either protein Cap coded in ORF2 of PCV2 or protein VP2 of PPV is the main structural protein constructed viral capsid, respectively. Both proteins have immunogenicity, so they are always used for vaccine development. Multivalent vaccines of PCV2 and PPV would be useful for reducing PCV2 and PPV in swine. With the development of a bicistronic mammalian expression vector or vectors exploiting internal ribosomal entry site (IRES) sequences from cytomegalovirus [14], poliovirus [15], and encephalomyocarditis virus (EMCV) [16-18], it is possible to co-express two proteins from a single messenger RNA (mRNA). In bicistronic mRNA, the first ORF upstream of IRES is translated by usual cap-dependent translation whereas the downstream ORF of IRES is translated in a cap-independent manner. ${ }^{19}$ This strategy has been used to develop bicistronic plasmid DNA expressing two proteins to induce immune responses $[17,20]$. A multivalent vaccine that could generate immunity in pigs against PPV and PCV2 without requiring multiple injections would be valuable.

*Corresponding author: Shangjin Cui, DVM, PhD, Division of Swine infectious Disease, State Key Laboratory of Veterinary Biotechnology, Harbin Veterinary Research Institute, No. 427, Maduanjie, Nangang District, Harbin, Heilongjiang 150001, People's Republic of China, Tel: 86451 85935093; Fax: 86451 82733132; E mail: cuishangjin@yahoo.com.cn

Received August 12, 2011; Accepted September 21, 2011; Published September 28, 2011

Citation: Lin W, Zhang Z, Cui S (2011) A Bicistronic DNA vaccine against Porcine Circovirus and Porcine Parvovirus. J Veterinar Sci Technol S1:001. doi:10.4172/2157-7579.S1-001

Copyright: () $2011 \mathrm{Lin} \mathrm{W}$, et al. This is an open-access article distributed under the terms of the Creative Commons Attribution License, which permits unrestricted use, distribution, and reproduction in any medium, provided the original author and source are credited. 
The first objective of this study was to develop a bicistronic vaccine against PPV and PCV2. The second objective was to compare the efficacy of bicistronic and monocistronic vaccines against these important viruses.

\section{Materials and Methods}

\section{Viruses and cells}

PCV2 and PPV were obtained from the Harbin Veterinary Research Institute of the Chinese Academy of Agricultural Sciences. PCV2 was propagated on PCV-free PK-15 cells, and PPV was propagated on PCV-free ST cells. Cells, all of which were obtained from the Harbin Veterinary Research Institute, were grown at $37^{\circ} \mathrm{C}$ under 5\% $\mathrm{CO}_{2}$ in 1640 medium, supplemented with $10 \%$ newborn calf serum (NBCS, Gibco BRL). For expression analysis, CHO cells were used for transfection.

\section{Construction of monocistronic and bicistronic DNA vaccines}

The PIRES bicistronic vector (Clontech, Palo Alto, CA, USA) was used to construct monocistronic and bicistronic DNA vaccines. This vector contained two multiple cloning sites (MCS) located on either side of the IRES from EMCV. The PCV2-ORF2 gene was subcloned from the recombinant plasmid pTargeT-RabG [17] into MCS-A of PIRES between the NheI and Mlu I restriction sites, and the recombinant plasmid pIRES-ORF2 was named monocistronic PCV2 DNA vaccine. Similarly, the monocistronic PPV vaccine pIRES-VP2 was constructed by subcloning the PPV-VP2 gene from the recombinant plasmid pETVP2 [21] into MCS-B of the PIRES vector between the XbaI and NotI restriction sites. The bicistronic pIRES-ORF2-VP2 DNA vaccine was constructed by subcloning both genes in one PIRES vector into MCS-A and MCS-B. The presence of PCV2-ORF2 and PPV-VP2 gene inserts was determined in both monocistronic and bicistronic DNA vaccines with restriction endonucleases.

\section{Analysis of bicistronic DNA vaccine co-expressing PCV2- ORF2 and PPV-VP2 in vitro}

Expression of recombinant plasmid pIRES-ORF2-VP2, pIRESORF2, pIRES-VP2, and the empty vector PIRES was analyzed in $\mathrm{CHO}$ cells after transfection using FuGENE HD transfection reagent (Roche) following manufacturer's instructions. Control cells were mocktransfected without plasmid. Cells were then grown for an additional $48 \mathrm{~h}$ in growth medium. Whole cell lysate of transfected and mocktransfected $\mathrm{CHO}$ cells were prepared by lysing the cell monolayer in SDS-PAGE sample buffer and separating the lysate on a $10 \%$ SDSPAGE gel. For Western blotting, proteins were transferred from the SDS-PAGE gel onto a nitrocellulose membrane and probed with either anti-PCV2 or anti-PPV polyclonal serum (obtained from the Harbin Veterinary Research Institute) at the 1:1000 ratio of dilution. The bound antibodies were detected using Anti-Rabbit IgG- HRP (sigma) at the 1:5000 ratio of dilution, and were visualized with DAB substrate solution.

\section{Large-scale preparation of plasmid DNA}

For preparation of the three DNA vaccine plasmids, a single colony of recombinant plasmids pIRES-ORF2-VP2, pIRES-ORF2, and pIRESVP2 were separately picked and inoculated in $10 \mathrm{~mL}$ of Luria Bertani (LB) broth containing $100 \mu \mathrm{g} / \mathrm{mL}$ ampicillin and incubated overnight at $37^{\circ} \mathrm{C}$. A $5-\mathrm{mL}$ volume of the overnight culture was added to 500 $\mathrm{ml}$ of $\mathrm{LB}$ plus ampicillin and grown overnight at $37^{\circ} \mathrm{C}$ with shaking. Bacterial cells were pelleted by centrifugation, and plasmid DNA was isolated using the EndoFree plasmid Giga kit (Qiagen, Cat\#12391) following manufacturer's instructions. The concentration of plasmid DNA was estimated spectrophotometrically. The plasmid DNAs used for immunization were ethanol precipitated and resuspended in PBS at a concentration of $100 \mu \mathrm{g} / \mathrm{mL}$. All DNA preparations were stored at $-20^{\circ} \mathrm{C}$ until used for immunization.

\section{Vaccination of mice with plasmid DNA vaccines}

Four groups of 4- to 6-week-old Balb/c mice (10 mice per group, and 20 mice for pIRES-ORF2-VP2 group) were immunized intramuscular with $100 \mu \mathrm{g}$ of one of the following samples: recombinant plasmid pIRES-ORF2-VP2, pIRES-ORF2, pIRES-VP2, and empty vector PIRES. An additional group of mice $(\mathrm{n}=10)$ was injected with $1 \mathrm{~mL}$ of PBS. And all the mice were given a total of 3 injections every two weeks. Mice were bled every week after the first immunization and assayed for PCV- or PPV-specific antibodies with ELISA. After 6 additional weeks, the cell immunity of mice was analyzed by the lymphocyte proliferation test and flow cytometry test. The average value and standard deviation for the level of immune responses within each group were analyzed using independent samples $t$-tests and are expressed as averages of two to three independent experiments. $p<0.05$ was considered significant.

\section{ELISA and Virus neutralization}

PPV antibody was tested with revised SVANOVIR ELISA kits (Svanvir Biotech AB, Uppsala, Sweden), and PCV antibody was tested with revised INGEZIM Circovirus IgG/IgM ELISA kits (Madrid, Spain), ELISA was performed according to protocol of kits, and revised according to Westenbrink et al. (1989). All washing steps were carried out five times with PBS-T buffer (0.05\% Tween 20 in PBS); all antigens were diluted in PBS, and antibodies were diluted in PBS-T buffer. The plates were washed and incubated for $1 \mathrm{~h}$ at room temperature with the appropriate dilution (1:100) of the mice serum and anti-mouse IgG-HRP (sigma) at the 1:5000 ratio of dilution. After the plates were washed with PBS-T, substrate solution was added, and the plates were incubated. The reaction was stopped after $10 \mathrm{~min}$ at room temperature, and the $\mathrm{OD}_{450} \mathrm{~nm}$ values were measured with a microtiter plate reader. The ELISA titres were defined as the reciprocal of the highest serum dilution positive in ELISA.

Virus neutralization (VN) test was performed using serum from each mouse as described in the OIE Manual [22]. The neutralizing antibody titer was calculated as the reciprocal of the highest serum dilution that neutralized $50 \%$ of the virus.

\section{T lymphocyte cell proliferation assay}

For the $\mathrm{T}$ cell proliferation assay, Balb/c mice were immunized as described above. The splenocytes from the immunized mice were harvested 2 weeks after the second booster injection, and extracted T lymphocyte cell with the lymphocyte separation medium (TBD) according to the instruction, and then cultured in 96 -well plates $\left(4 \times 10^{5}\right.$ splenocytes/well) with $200 \mu \mathrm{L}$ of RPMI 1640 containing $10 \%$ FBS, in the presence of alive PPV, alive PCV, or concanavalin A (Con A). The virus was used as the experimental stimulant whereas Con A was considered as the positive control. In parallel, the splenocytes were incubated with the medium as the negative control. The stimulation continued at $37^{\circ} \mathrm{C}$ for $80 \mathrm{~h}$, and then Con A was added to each well for the last 5h of incubation. After the splenocytes were cultured, $\mathrm{OD}_{450} \mathrm{~nm}$ values were measured with a microtiter plate reader. The stimulation index (SI) was determined according to the formula: $\mathrm{SI}=($ experimental count spontaneous count)/spontaneous count. 
Citation: Lin W, Zhang Z, Cui S (2011) A Bicistronic DNA vaccine against Porcine Circovirus and Porcine Parvovirus. J Veterinar Sci Technol S1:001. doi:10.4172/2157-7579.S1-001

\section{Flow cytometry analysis of levels of CD4+ and CD8+ T cells}

Mice that survived the challenge were sacrificed on day 15 postchallenge, and splenocytes were prepared following established protocols [23]. Lymphocytes were stimulated with PPV and PCV2 about $16 \mathrm{~h}$, and then harvested to prepare for cellular suspensions at a final concentration of $5 \times 10^{6}$ per millilitre. The cells were stained with PerCP-conjugated rat anti-mouse CD4+ and phycoerythrin (PE)conjugated rat anti-mouse CD8+ antibodies (BD Pharmingen, San Diego, CA, USA). The number of cells of CD4+ and CD8+ T cells were determined by flow cytometry on a BD FACS Calibur Station (BD Immunocytometry Systems, San Jose, CA, USA), and data were analyzed with Flowjo 4.2 software (Tree Star, Ashland, OR).

The average value and standard deviation for the level of immune responses within each group were analyzed using independent samples $t$-tests and are expressed as averages of two to three independent experiments. $\mathrm{p}<0.05$ was considered significant.

\section{Safety of the bicistronic DNA vaccine}

For analysis of safety, the genomic DNA was extracted from the organs (heart, liver, spleen, lung, kidney, and brain) of mice treated with the recombinant plasmid pIRES-VP2-ORF2, and the recombinant plasmid genes were amplified by PCR to determine whether the recombinant genes had integrated into the mouse genome.

\section{Results}

\section{Construction of a bicistronic DNA vaccine co-expressing PCV2-ORF2 and PPV-VP2}

The monocistronic and bicistronic DNA vaccine plasmids encoding PCV2-ORF2 and PPV-VP2 were constructed using the bicistronic vector PIRES (Clontech) as shown in Figure 1. Westernblot indicated that the bicistronic DNA vaccine plasmid pIRES-ORF2VP2 co-expressed both proteins in transfected cells (Figure 2). The cell lysate contained a $\sim 32-\mathrm{kDa}$ protein that reacted with anti-PCV immune serum in Western-blot. The cell lysate also contained a $\sim 64-$ $\mathrm{kDa}$ protein that reacted with anti-PPV-specific hyperimmune serum. The monocistronic DNA vaccine plasmid pIRES-ORF2 expressed the protein Cap, and the monocistronic DNA vaccine plasmid pIRES-VP2 expressed the protein VP2. There were no corresponding proteins in cell lysate from control mock-transfected $\mathrm{CHO}$ cells or in cell lysate from $\mathrm{CHO}$ cells transfected with plasmid PIRES.

PIRES
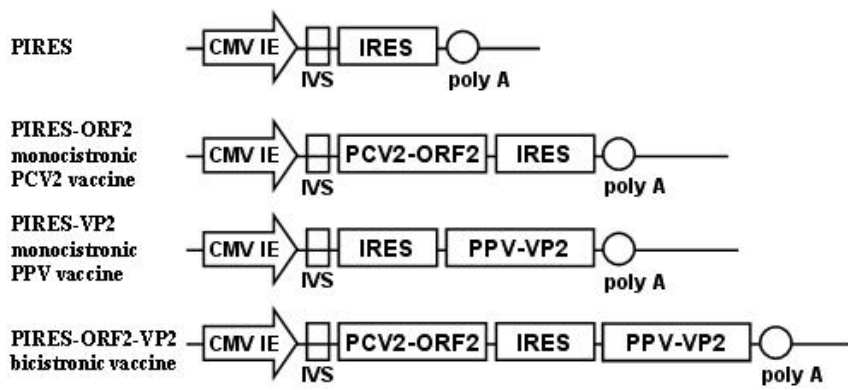

Figure 1: Schematic diagram of the monocistronic and bicistronic DNA vaccines.

The transcriptional control units and gene sequences are shown: CMV $\mathrm{IE}$, cytomegalovirus immediate early promoter/ enhancer sequence; IVS, intervening sequence (synthetic intron); IRES, internal ribosome entry site polyA, SV40, polyadenylation signal; PCV2-ORF2, porcine circovirus type 2 ORF2 gene; PPV-VP2, porcine parvovirus VP2 gene.
(A)

$\begin{array}{llllll}M & 1 & 2 & 3 & 4 & 5\end{array}$

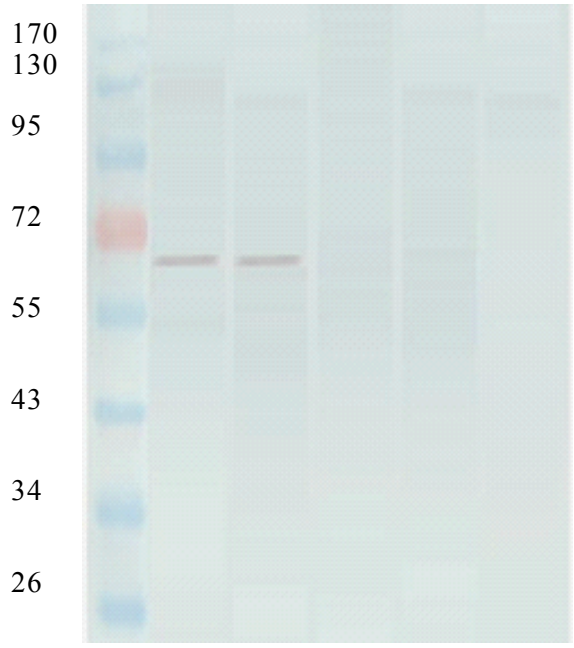

(B)

$\begin{array}{llllll}M & 1 & 2 & 3 & 4 & 5\end{array}$

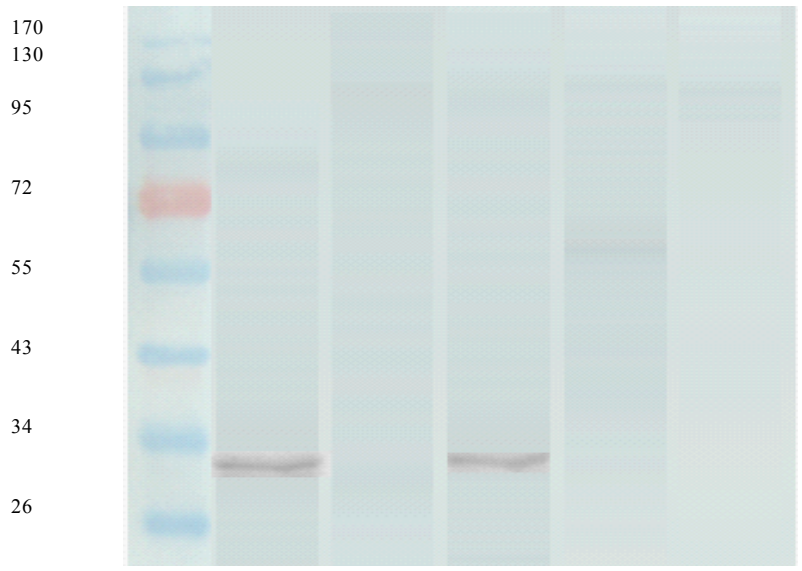

Figure 2: Western-blot detection of (a) PPV-VP2 expression and (b) PCV2ORF2 expression.

a: M: Marker; 1: pIRES-VP2-ORF2 group; 2: pIRES-VP2 group; 3: pIRESORF2 group; 4: PIRES group; 5: CHO cell control group.

b: M: Marker; 1: pIRES-VP2-ORF2 group; 2: pIRES-VP2 group; 3: pIRESORF2 group; 4: PIRES group; 5: CHO cell control group.

Induction of anti-PCV and anti-PPV responses in mice immunized with monocistronic and bicistronic DNA vaccines

After vaccine expression was characterized in vitro, the bicistronic DNA vaccine, monocistronic DNA vaccines, and control PIRES plasmid were injected into mice. All mice receiving either monocistronic or bicistronic DNA vaccine showed seroconversion and developed significant ELISA antibody responses against both PCV and PPV (Figure 3). There was no specific antibody response in unimmunized mice or in the mice receiving PIRES plasmid. ELISA antibody titres elicited by monocistronic and bicistronic DNA vaccines were similar.

The virus neutralizing capacity of induced antibody responses in immunized mice was evaluated in virus neutralizing assay of sera against PPV and PCV in vitro. Results demonstrated that all mice immunized with either monocistronic or bicistronic DNA vaccines 
Citation: Lin W, Zhang Z, Cui S (2011) A Bicistronic DNA vaccine against Porcine Circovirus and Porcine Parvovirus. J Veterinar Sci Technol S1:001. doi:10.4172/2157-7579.S1-001

exhibited VN antibody response against PPV and PCV on day 28 post immunization with no significant difference (Table 1). And 0.5 VN titres against PPV and PCV could produce protective capability.

\section{Cellular immune responses elicited by DNA vaccine immunization}

Whether the monocistronic and bicistronic DNA vaccine induced $\mathrm{T}$ cell responses was first investigated by the splenocyte proliferation assay. Each mouse was immunized with $100 \mu \mathrm{g}$ of recombinant plasmids, empty vector PIRES, and PBS as described above. At 2 weeks

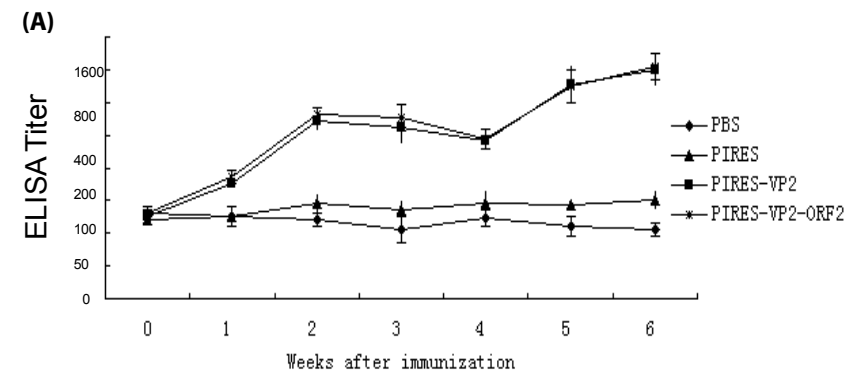

(B)

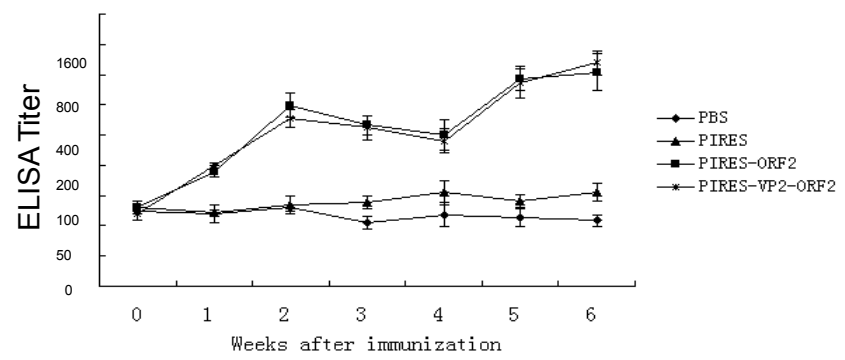

Figure 3: Specific antibody response in mice to (A) PPV and (B) PCV2.

(A)

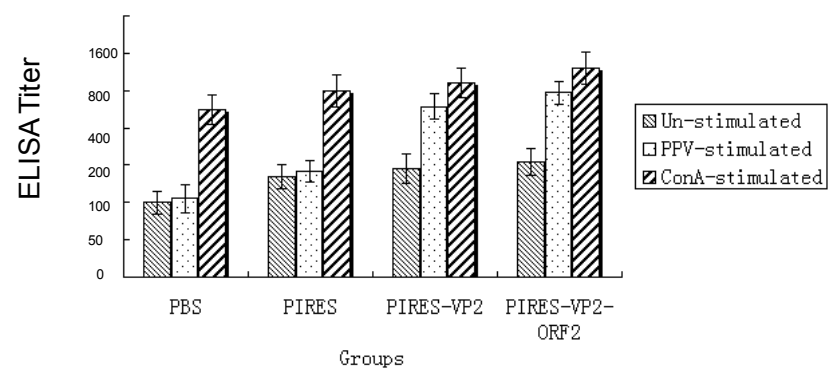

(B)

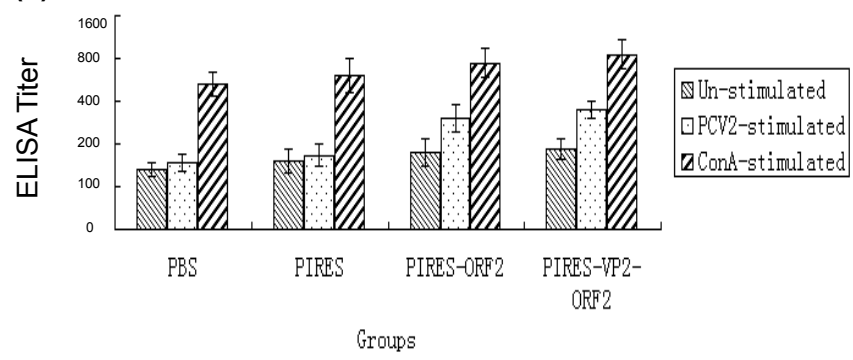

Figure 4: Proliferative response of splenic lymphocytes against (A) PPV and ConA; and (B) PCV2 and ConA.

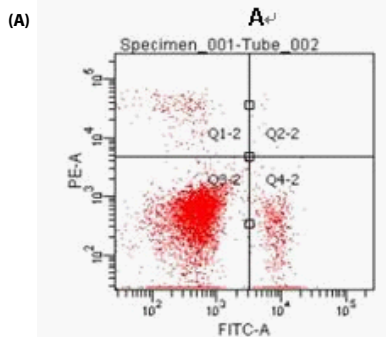

A

(B)
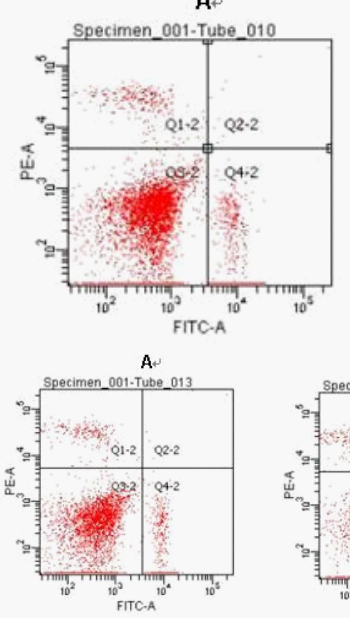

A

(D)

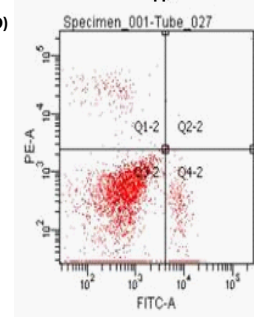

A

(E)

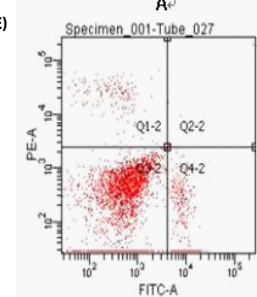

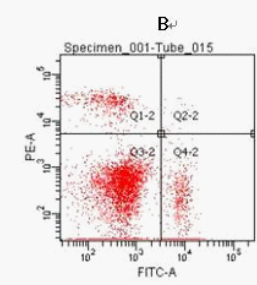

B

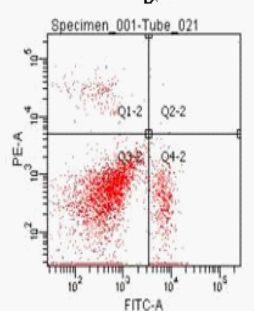

B

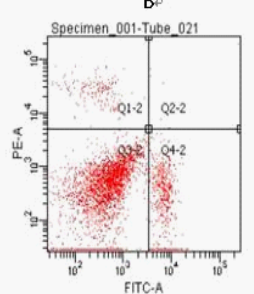

B

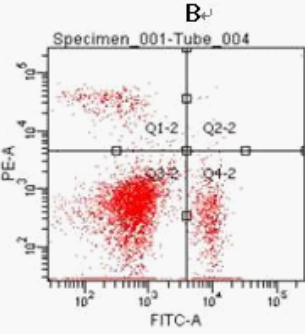

B

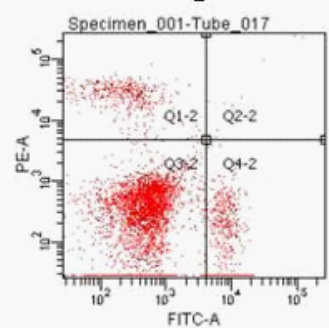

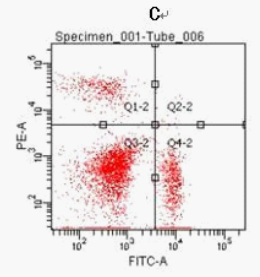

C.

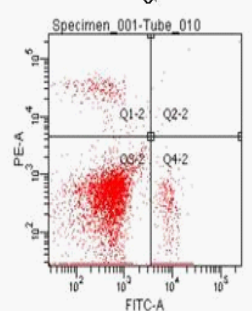

C

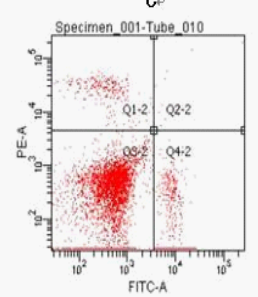

Figure 5: Proliferation of CD4+ and CD8+ cells after stimulation by PPV or PCV2 in mice that were vaccinated with (a) pIRES-VP2, (b) pIRES-ORF2, (c) pIRES-VP2-ORF2, (d) PIRES, or (e) PBS.

a: A: Un-stimulated B: PPV-stimulated.

b: A: Un-stimulated B: PCV-stimulated.

c: A: Un-stimulated B: PPV-stimulated C: PCV2-stimulated.

d: A: Un-stimulated B: PPV-stimulated C: PCV2-stimulated.

e: A: Un-stimulated B: PPV-stimulated C: PCV2-stimulated.

post-booster injection, the splenocytes were harvested and stimulated with PCV and PPV virus. The lymphocyte proliferation test showed that the level of spleen lymphocyte proliferation increased in mice treated with recombinant plasmid but not in mice treated with empty vector PIRES or PBS (Figure 4). The SI values of Con A-stimulated splenocytes from the DNA vaccine groups were similar to those from the empty vector PIRES group, thereby indicating comparable 


\begin{tabular}{|l|l|l|l|l|l|l|l|l|l|l|l|l|l|}
\hline & Negative control & Vaccine control & VP2-CAP & VP2 & CAP & PBS control & PIRES control & 0 & 0 \\
\hline PPV & 0 & 16 & 32 & 32 & 0 & 32 & 0 \\
\hline PCV2 & 0 & 32 & 32 & 0 & 0 \\
\hline
\end{tabular}

Table 1: Detection of antibody against PPV and PCV2 in mice by neutralization tests.

health and number of all splenocytes used in this experiment. Upon stimulation with virus, the SI values of the bicistronic DNA vaccine group was approximately two times that of the PIRES lysate group. The SI values were statistically similar $(p>0.05)$ for the bicistronic DNA vaccine plasmid group and the monocistronic DNA vaccine groups.

\section{Levels of CD4+ and CD8+ T cells}

The flow cytometry test indicated that the levels of CD4+ and CD8+ cells were increased by 10.7 and 5.3\%, respectively, after PPV stimulus in the pIRES-VP2 group (Figure $5 \mathrm{a}$ ); by 10.1 and $4.8 \%$, respectively, after PCV stimulus in the pIRES-ORF2 group (Figure 5b); by 12.7 and $3.9 \%$, respectively, after PPV stimulus in the pIRES-VP2-ORF2 group (Figure 5c); and by 12.5 and $4.8 \%$, respectively, after PCV stimulus in the pIRES-VP2-ORF2 group (Figure 5d). There was no obvious change in levels of CD4+ and CD8+ after PPV or PCV2 stimulus in the PIRES group. The results of this immunity test showed that the constructed recombinant plasmid could produce humoral and cellular immune responses in mice.

\section{Safety of bicistronic DNA vaccine}

No lesions were present on the organs of mice treated with the DNA vaccines, and the recombinant plasmid pIRES-VP2-ORF2 did not integrate into the mouse chromosome by PCR (data not shown).

\section{Discussion}

Between 1990 and 1993, the administration of plasmid DNA encoding a specific protein antigen was shown to induce expression, antibody response against protein, and protection against infection by various pathogens [10]. Since then, the efficacy of DNA vaccines in small and large animal models of infectious diseases and cancer has been tested and reported in more than 1000 publications, and the results were frequently satisfactory [24]. Several clinical trials of DNA vaccines against HIV, malaria, hepatitis $B$, and herpes virus and in cancer therapy are under evaluation.

In this study, we have constructed a bicistronic DNA vaccine, pIRESORF2-VP2, that utilizes IRES sequences from encephalomyocarditis virus and encodes PCV2-ORF2 and PPV-VP2. This bicistronic DNA vaccine co-expressed both proteins (i.e., PCV2 Cap and PPV VP2) in vitro as demonstrated by Western blot. When the bicistronic DNA was injected in mice, all immunized mice showed seroconversion for both antigens. An immunity test showed that the constructed recombinant plasmid could produce both humoral and cellular immune responses in mice. The ELISA antibody response to the bicistronic DNA was similar to the responses to the corresponding monocistronic DNA vaccines against PCV2 and PPV. This indicated that both proteins were expressed in a fully immunogenic form and presented to the immune cells independently without one masking the effect of the other. The level of the cellular immune response was measured by flow cytometry and by $\mathrm{T}$ lymphocyte cell proliferation assay; the results were the same as with ELISA.

Several reports have indicated that the protein downstream to the IRES sequence is expressed less than the protein upstream of the IRES sequence element, perhaps because of difference in translational efficiency or because large quantities of the first protein interfere with synthesis and processing of the other protein [25]. Further, Manoj et al. have also reported reduced immune response to the gene downstream of IRES in a bicistronic DNA vaccine plasmid due to antigen competition for immune cells [14]. In contrast, our study found equivalent expression of PPV-VP2 protein (from the gene cloned downstream of IRES) and the PCV2-ORF2 (from the gene cloned upstream of IRES) in pIRES-ORF2-VP2-transfected CHO cells. PCV2-ORF2 also was expressed when the positions of the cloned genes were reversed, indicating no effect of gene order on protein expression (data not shown). Although our assay was not quantitative (we did not determine the amount of protein expressed in each cell), the presence of both proteins in each cell was shown by flow cytometric analysis. This indicated that all transfected cells were expressing both proteins. Further, the immune response against PPV-VP2 in mice treated with the bicistronic DNA was comparable to the immune response induced by the monocistronic PPV vaccine. After immunization of monocistronic and bicistronic DNA vaccines in mice, seroconversion for both the antigens was found with no significant difference, indicating no antigenic competition and suggesting that expression was similar for the two proteins. In an earlier study, protection against rabies infection was similar for mice treated with a bicistronic DNA rabies vaccine and a monocistronic rabies vaccine [10].

Short of a large animal model in this experiment now and swine experiment will go on next time. And the CD8 cells functional CTLs, in addition to data on intracellular IFN $-\gamma$ stain, in vitro cell lysis assays (Molecular Probes LIVE/DEAD Viability/Cytotoxicity Kit) of PPV and PCV2-infected swine testicular and pig kidney cell lines, respectively), will be considered.

In conclusion, this study demonstrates that a bicistronic DNA vaccine was capable of inducing a virus-neutralizing antibody response against PCV2 and PPV that was similar to the antibody response induced by monocistronic DNA vaccines. There was no evidence of competition between the two expressed antigens for antibody production.

\section{Acknowledgments}

We thank Dr. Huaji Qiu for helpful technical suggestions and critical comments on the manuscript. This work was supported by the Chinese National 10th FiveYear Science and Technology Plan (grant 2004 BA519A01) and National Hightech R\&D Program (2007AA100606).

\section{References}

1. Clark EG (1997) Post-weaning multisystemic wasting syndrome. Proceedings of the American Association of Swine Practitioners. 499-501.

2. Harding JS (1997) Recognizing and diagnosing postweaning multisystemic wasting syndrome (PMWS). J Swine Health Prod 5: 201-203.

3. Segalés J, Allan GM, Domingo M (2005) Porcine circovirus diseases. Anim Health Res Rev 6: 119-142.

4. Mengling WL (1999) Porcine parvovirus. In: Straw SD, Allaire WL. Mengeling DJ, eds. Diseases of Swine. 8th ed. lowa. lowa State University Press. 187200.

5. Choi C, Chae C (2000) Distribution of porcine parvovirus in porcine circovirus 2 - infected pigs with postweaning multisystemic wasting syndrome as shown by in-situ hybridization. J Comp Pathol 123: 302-305

6. Kim J, Chung HK, Jung T, Cho WS, Choi C, et al. (2002) Postweaning multisystemic wasting syndrome of pigs in Korea: prevalence, microscopic lesions and coexisting microorganisms. J Vet Med Sci 64: 57-62. 
Citation: Lin W, Zhang Z, Cui S (2011) A Bicistronic DNA vaccine against Porcine Circovirus and Porcine Parvovirus. J Veterinar Sci Technol S1:001. doi:10.4172/2157-7579.S1-001

Page 6 of 6

7. Ellis JA, Bratanich A, Clark EG (2000) Coinfection by porcine circoviruses and porcine parvovirus in pigs with naturally acquired postweaning multisystemic wasting syndrome. J Vet Diagn Invest 12: 21-27.

8. Kennedy S, Moffett F, McNeilly B, Meehan B, Ellis J, et al. (2000) Reproduction of lesions of postweaning multisystemic wasting syndrome by infection of conventional pigs with porcine circovirus type 2 alone or in combination with porcine parvovirus. J Comp Pathol 122: 9-24.

9. Krakowka S, Ellis J, McNeilly F, Waldner C, Allan G (2005) Features of porcine circovirus-2 disease: correlations between lesions, amount and distribution of virus, and clinical outcome. J Vet Diagn Invest 17: 213-222.

10. Westenbrink F, Straver PJ, Kimman TG., de Leeuw PW (1989) Development of a neutralising antibody response to an inoculated cytopathogenic strain of bovine virus diarrhoea virus. Vet Rec 125: 262-265.

11. Wolff JA, Malone RW, Williams P (1990) Direct gene transfer into mouse muscle in vivo. Science 47: 1465-1468.

12. Liu MA (1995) Overview of DNA vaccines. Ann N Y Acad Sci 772: 15-20.

13. Liu MA (2003) DNA vaccines: a review. J Int Med 253: 402-410.

14. Manoj S, Babiuk LA, van Drunen Littel-van den Hurk S (2003) Immunization with a dicistronic plasmid expressing a truncated form of bovine herpes-1 glycoprotein $D$ and the amino-terminal subunit of glycoprotein $B$ results in reduced gB-specific immune responses. Virol 313: 296-297.

15. Kauder SE, Racaniello VR (2004) Poliovirus tropism and attenuation are determined after internal ribosome entry. J Clin Invest 113: 1743-1753.

16. Ghattas IR, Sanes JR, Major JE (1991) The encephalomyocarditis virus internal ribosomal entry site allows efficient coexpression of two genes from a recombinant provirus in cultured cells and in embryos. Mol Cell Biol 11: 58485859 .
17. Henke A, Rohland N, Zell R, Wutzler P (2006) Co-expression of interleukin-2 by a bicistronic plasmid increases the efficacy of DNA immunization to prevent influenza virus infections. Intervirol 49: 249-252.

18. Rainczuk A, Scorza T, Spithill TW, Smooker PM (2004) A bicistronic DNA vaccine containing apical membrane antigen 1 and merozoite surface protein $4 / 5$ can prime humoral and cellular immune responses and partially protect mice against virulent Plasmodium chabaudi adami DS malaria. Infect Immun 72: $5565-5573$.

19. Hellen CT, Sarnow $P$ (2001) Internal ribosome entry sites in eukaryotic mRNA molecules. Genes Dev 15: 1593-1612.

20. Gurtu V, Yan G, Zhang G (1996) IRES bicistronic expression vectors for efficient creation of stable mammalian cell lines. Biochem Biophys Res Commun 229: 295-298.

21. Qi T, Cui S (2009) Expression of porcine parvovirus VP2 gene requires codon optimized E. coli cells. Virus Genes 39: 217-222.

22. OIE (2010) OIE, W.O.f.A.H. (2010) OIE Terrestrial Manual. Chapter Bovine Viral Diarrhoea. (2008) Paris.

23. Ye L, Bu Z, Vzorov A, Taylor D, Compans RW, et al. (2004) Surface stability and immunogenicity of the human immunodeficiency virus envelope glycoprotein role of the cytoplasmic domain. J Virol 7: 13409-13419.

24. Kutzler MA, Weiner DB (2008) DNA vaccines: ready for prime time? Nat Rev Genet 9: 776-788.

25. Kim D, Kigawa T, Choi C, Yokoyama S (2004) A Highly Efficient Cell-Free Protein Synthesis System from Escherichia coli. Europ J Biochem 239: 881- 886. 